\title{
PREMIĖRES OBSERVATIONS SUR LA TRUITE (SALMO TRUTTA L.) DANS LE REDON, UN PETIT AFFLUENT-FRAYERE DU LAC LÉMAN
}

\author{
A. Champigneulle (1), M. mélhaoui (2), G. maisse (3), J.L. BAGLINiÉre (3), \\ C. GILLET (1), D. GERDEAUX (1)
}

avec la collaboration technique de P. LAURENT (1) et J. ESCOMEL (1)

(1) Station d'Hydrobiologie Lacustre, I.N.R.A., BP 11 F, 75, avenue de Corzent - 74203 THONONLES-BAINS, France.

(2) Université Mohammed $1^{\mathrm{e}}$, Faculté des Sciences, Département de Biologie - OUJDA, Maroc.

(3) Station de Physiologie et d'Écologie des Poissons. I.N.R.A. - 35042 RENNES Cedex, France.

\section{RÉSUME}

La reproduction de la truite commune ( $S a l m o$ trutta $L$.) a été suivie trois années consécutives du début novembre à la mi-janvier, dans un petit affluent du Lac Léman. Le suivi a été réalisé en utilisant deux techniques complèmentaires: la pêche électrique et le comptage des frayères. L'activité de fraie des truites lacustres est comparée à celle des truites sédentaires. L'étude précise les caractéristiques des populations en place avant la fraie, ainsi que celles des géniteurs de truite de lac. Les mouvements des juvéniles sont sommairement étudiés.

\section{PRELIMINARY STUDY OF THE BROWN TROUT (SALMO TRUTTA L.) IN THE RIVER REDON, A SMALL TRIBUTARY OF LAKE LEMAN}

\section{SUMMARY}

The spawning of brown trout (Salmo trutta L.) was studied during three successive winters between the beginning of November and mid-January, in a small tributary of lake Leman. The survey was realized by using two complementary techniques : electrofishing and the counting of redds. The spawning activity is compared between sedentary and migrating trouts. The study describes the characteristics of sedentary trouts just before the spawning season and the one of migrating trout spawners. The movements of juveniles are briefly studied.

\section{INTRODUCTION}

Dans beaucoup de lacs européens, les populations naturelles de truite (Salmo trutta L.) de lac sont menacées (LELEK, 1980). Comme dans le cas du lac de Constance (RUHLE et al., 1984), c'est la phase en rivière (reproduction et production de juvéniles) du cycle de vie qui est généralement fragilisée du fait de la dégradation des milieux: pollutions, barrages, prises d'eau accentuant les étiages.

Dans le cas du lac Léman (58.230 ha), les captures totales annuelles (pêche amateur et pêche professionnelle) restent importantes puisqu'elles ont varié entre 15 et $35 \mathrm{t}$ lors des dix dernières années. Les captures sont essentiellement composées de truite commune; la truite arc-en-ciel (Salmo gairdneri $\mathrm{R}$.) représente moins de $5 \%$ des prises. Les relâchers d'alevins et de truitelles fario ont été intensifiés (actuellement 1 à 1,5 millions/an), mais leur impact réel n'a pas encore été quantifié. Des recherches récentes ont porté sur la biologie de la truite au Léman (MELHAOUI, 1985 ; BUTTIKER et MATTHEY, 1986; BUTTIKER et al., 1987; CHAMPIGNEULLE, 1987). Trois thèmes principaux sont abordés:

- les caractéristiques des captures au lac.

- l'efficacité des divers modes de repeuplement.

- la fraie et la production de juvéniles dans les affluents.

Le présent article porte sur le dernier thème. Il vise principalement à décrire les caractéristiques des géniteurs et de la fraie des truites de lac (truites ayant eu une phase de croissance en lac) et des truites sédentaires (truites ayant effectue tout leur cycle biologique en milieu rivière) dans un petit affluent du Léman. Le suivi de la reproduction a été complété par une étude de la population en place visant notamment à réaliser une première approche des caractéristiques des juvéniles (densité, taille, mouvements). 


\section{MATÉRIEL et MÉthodes}

\subsection{Localisation de l'Étude}

Les travaux ont été menés trois années consécutives de 1983 à 1985 sur le cours principal du Redon, un petit affluent de la rive française du Léman (Fig. 1). Les milieux étudiés ont été présentés en détail par MELHAOUI (1985). Leurs caractéristiques principales sont résumées dans le tableau I.

Dans l'étude, trois zones sont considérées sur le cours principal du Redon (fig. 1)

\begin{tabular}{|c|c|c|}
\hline \multirow[t]{2}{*}{ Zone } & \multicolumn{2}{|c|}{ Distance à l'embouchure } \\
\hline & aval & amont \\
\hline $\begin{array}{l}A \\
1 \\
B\end{array}$ & $\begin{array}{c}0 \\
1.600 \\
3.500\end{array}$ & $\begin{array}{l}1.600 \mathrm{~m} \\
3.500 \mathrm{~m} \\
4.100 \mathrm{~m}\end{array}$ \\
\hline
\end{tabular}

Les zones $A$ et I sont ouvertes à la pêche (début mars à début octobre) alors que la zone $B$ constitue la partie aval d'une réserve de pêche. La limite amont de la zone A est constituée par une carrière que le Redon traverse, canalisé sur $50 \mathrm{~m}$ dans des buses (diamètre: $1 \mathrm{~m}$, vitesse du courant en débit moyen $:>4 \mathrm{~m} / \mathrm{s}$ ) constituant un obstacle pratiquement infranchissable.

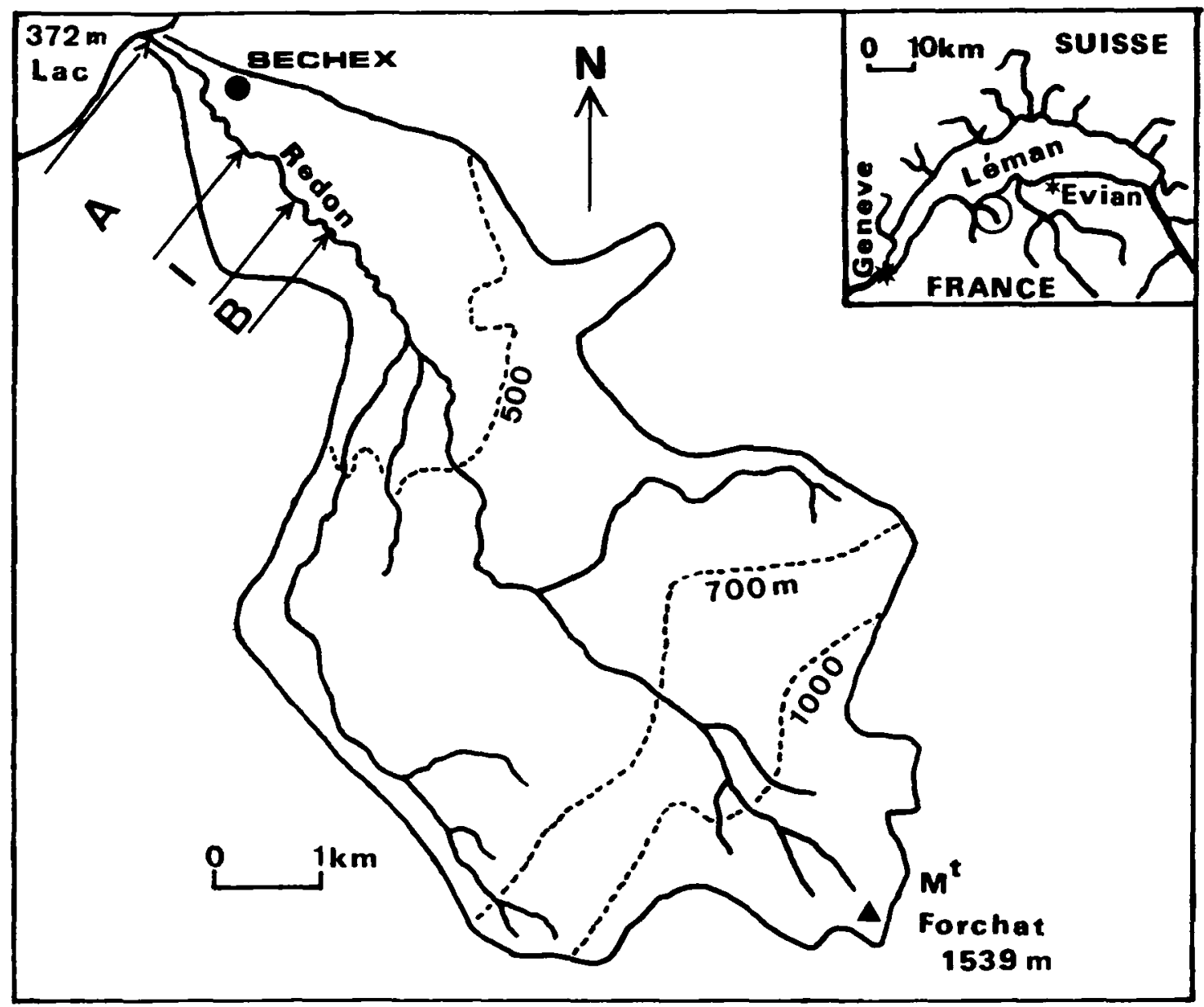

Figure 1 : Le bassin versant du Redon. Localisation des zones (A, B, I) d'étude de la fraie de la truite.

Figure 1 : Location of the River Redon and of the sections $(A, B, I)$ studied during trout reproduction. 
Tableau 1 : Caractéristiques du Lac Léman et du Redon.

Table I : Characteristics of Lake Leman and River Redon.

\begin{tabular}{|c|c|}
\hline LAC LEMAN & RE[DON \\
\hline 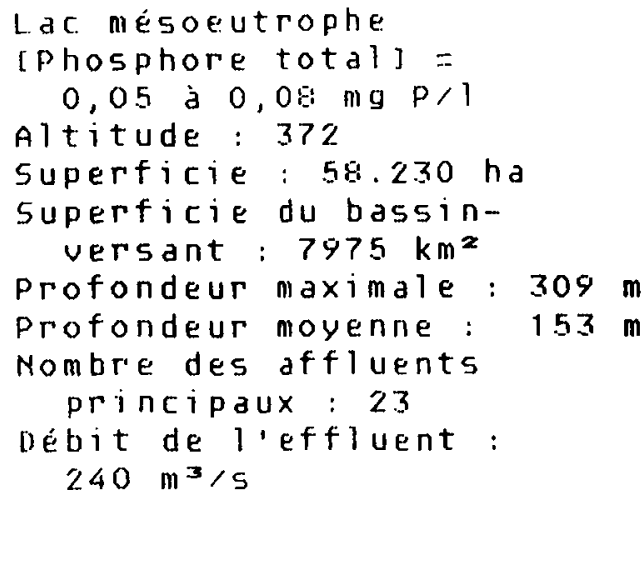 & 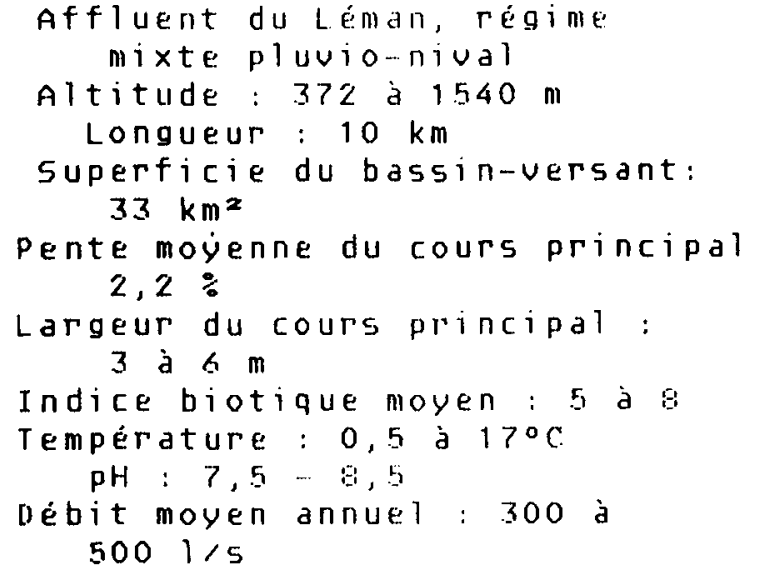 \\
\hline
\end{tabular}

\subsection{Population en place avant la fraie et caractéristiques des géniteurs sédentaires}

Des inventaires automnaux ont été réalisés par pêche électrique (2 ou 3 passages) en fin octobre 1984 et 1985 dans un secteur $\left(A_{1}\right)$ de la zone $A$ et dans 2 secteurs $\left(B_{1}\right.$ et $\left.B_{2}\right)$ de la zone $B$. Les estimations de densité, obtenues par la méthode DE LURY ou CARLE et STRUB (in GERDEAUX, 1987) sont très proches du nombre de truites capturées puisque ce dernier dépassait $95 \%$ du nombre estimé dans chacun des inventaires réalisés. Les secteurs, choisis comme étant représentatifs des zones $A$ et $B$, ont été délimités par des filets au moment des pêches. Les caractéristiques des secteurs sont décrites dans le tableau II. Les truites ont été mesurées (longueur à la fourche: LF et longueur totale: $(T)$ et des écailles ont été prélevées pour la détermination de l'âge.

Les géniteurs potentiels capturés ont été sexés: les mâles, par obtention de laitance et les femelles en vitellogénèse par sérodiagnostic selon la méthode décrite par LE BAIL et al., (1981) qui permet de déterminer précocement toutes les femelles reproductrices.

\subsection{Caractéristiques des géniteurs d'origine lacustre}

L'ensemble de la zone $A$ a été pêché à l'électricité (1 passage d'efficacité moyenne estimée à $70-80 \%$ ) : 9 fois du $9-11-83$ au $28-1-84 ; 11$ fois du 9-11-84 au 16-1-85 et 10 fois du 30-10-85 au 20-1-86. Par ailleurs, les principaux pools des zones $B$ et $I$ ont également été sondés trois fois au cours de chaque période de reproduction afin de rechercher l'éventuelle présence de géniteurs de truite de lac en amont de la zone $A$.

A chaque sondage, seules les truites capturées ayant une longueur totale (LT) supérieure à $25 \mathrm{~cm}$ ont été examinées. Les géniteurs ont été sexés, mesurés, marqués (Carlin) puis relâchés à proximité du lieu de capture. Des écailles ont été prélevées pour détermination de l'âge et pour la recherche des zones de croissance forte typiques de la phase lacustre. Bien que systématiquement vérifié, ce dernier critère n'est apparu indispensable (dans le cas de la présente étude) à la distinction truite de lac - truite sédentaire que dans un très faible nombre de cas, pour des truites de taille inférieure à $30 \mathrm{~cm}$. Dans la quasi totalité des cas, les truites de lac étaient déjà reconnaissables à la capture grâce à la livrée argentée de leur robe et/ou à leur très grande taille.

\section{4. Étude de la fraie et des frayères}

Le creusement des frayères a été régulièrement suivi sur les zones $A$ et $B$. En fin de période de reproduction, la zone $I$ et l'amont de la zone $B$ ont été parcourus afin d'y rechercher l'éventuelle présence de frayères de truite de lac. Sur les zones A et B les frayères ont été comptées et répertoriées par 6,11 et 15 passages, respectivement, au cours de la fraie de 1983, 84 et 85 . L'activité de fraie a été représentée graphiquement par le nombre cumulé d'apparitions de nouvelles frayères. La période d'activité maximale de fraie a été définie comme étant celle pendant laquelle le pourcentage cumulé des frayères recensées est passé de 10 à $90 \%$. Pendant le suivi de la reproduction, la température de l'eau a été mesurée dans le Redon et en bordure du lac. Les variations de niveau ont été enregistrées grâce à un limnigraphe placé à l'aval du Redon. 
Tableau II : Caractéristiques des secteurs du Redon inventoriés par péche électrique. Les différents habitats ont été caractérisés selon la méthode décrite par CHAMPIGNEULLE, 1978.

Table II : Characteristics of the different sections of the River Redon studied by electrofishing. The characteristics of the habitat are described according to the method of CHAMPIGNEULLE, 1978.

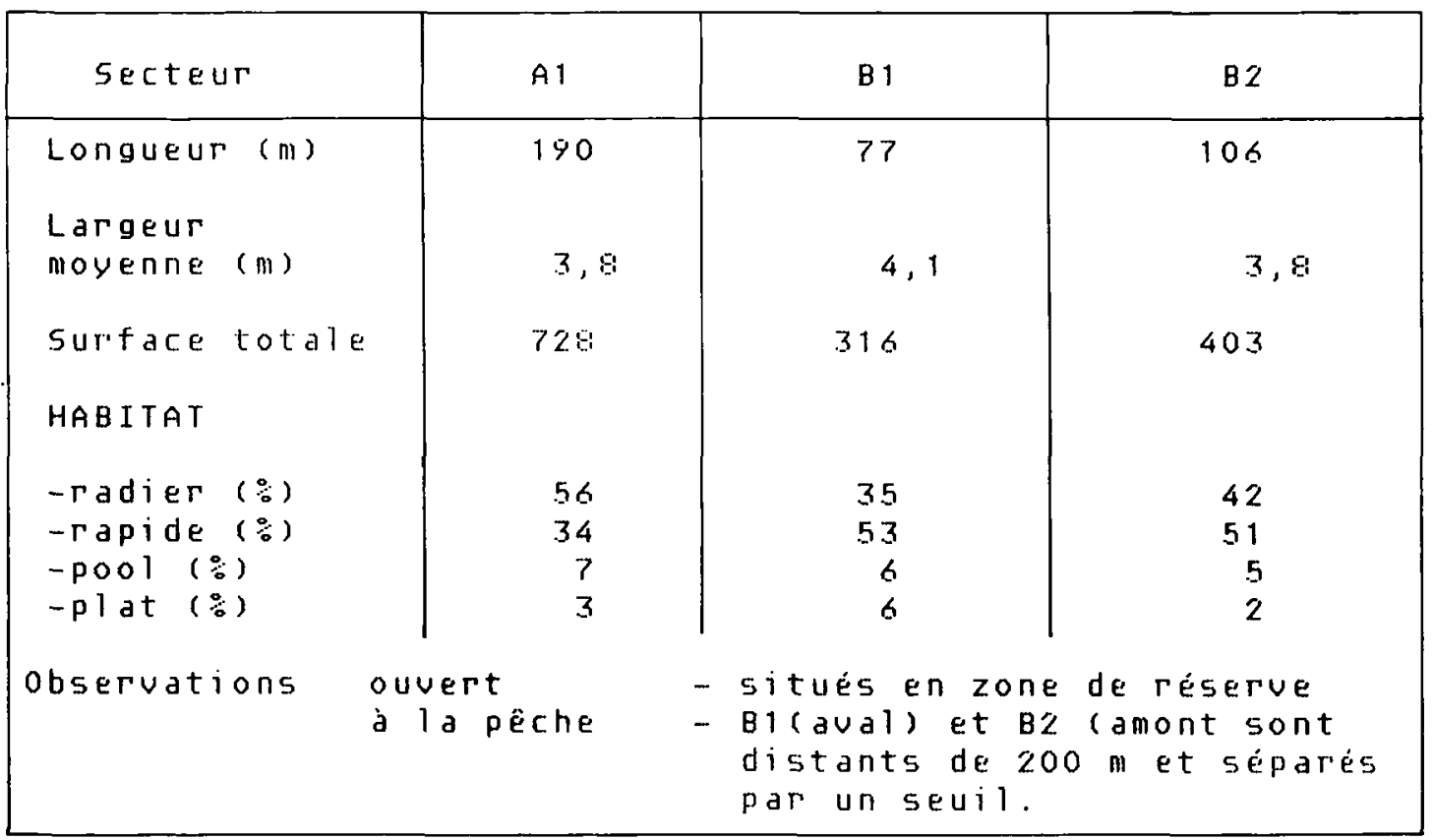

La distinction entre frayères de truites de lac et frayères de truites sédentaires a été facilitée par la très bonne connaissance de la répartition, dans l'espace et dans le temps, des deux types de géniteurs. L'absence de géniteurs de truite de lac dans la zone $B$ a permis d'attribuer les frayères de cette zone aux truites sédentaires. Sur la zone (A) commune de fraie, la détermination de l'appartenance des frayères a été réalisée de la façon suivante:

- en début de fraie, lorsqu'aucune femelle de truite de lac n'a encore été capturée, toutes les frayères ont été attribuées aux truites sédentaires.

- lorsque les deux formes de géniteurs femelles étaient présentes, une procédure plus complexe a du être adoptée pour limiter les risques d'erreur. Tenant compte des caractéristiques des frayères sùres de truites sédentaires, des fortes différences de taille entre les deux types de géniteurs ( $<30 \mathrm{~cm}$ pour les femelles sédentaires et $30-80 \mathrm{~cm}$ pour les femelles lacustres) et des données bibliographiques sur les relations taille des femelles - taille des frayères (OTTAWAY et al., 1981), les plus petites $(<80 \mathrm{~cm})$ et les plus grandes $(100-200 \mathrm{~cm})$ frayères ont été respectivement attribuées aux truites sédentaires et aux truites lacustres. Pour les frayères de taille intermédiaire $(80-100 \mathrm{~cm})$, la bonne attribution a pu être confirmée par l'observation des géniteurs sur les frayères et/ou par l'examen de la taille des cufs déposés (obtention par grattage du dôme et recueil des œufs dans un filet de dérive placé en aval). La taille des œufs des femelles sédentaires $(\mathrm{L}<30 \mathrm{~cm}$ ) variait de 3,7 à $4,5 \mathrm{~mm}$ alors que celle des truites de lac était de 4,9 à $6 \mathrm{~mm}$ (MELHAOUI, 1985).

\subsection{Caractéristiques des juvéniles $0+$ et $1+$ et de leur devenir}

Les caractéristiques (densité, taille) des juvéniles issus du recrutement naturel ont été décrites à partir des données d'inventaire de fin octobre 1984 et 1985 . Les truitelles 0 + de repeuplement, marquées ( 2 pelviennes coupées) et dispersées sur les zones A et l en 1983 (6.000 en août) et 1985 (5.800 en juin), ne sont pas prises en compte dans la présente étude. Le rapport des sexes a été calculé par autopsie sur un échantillon de 58 truitelles de la zone A.

Une première approche du devenir des juvéniles a été réalisée par une expérience de marquage et par piégeage partiel. Lors des inventaires de fin octobre 1984, tous les juvéniles des secteurs $A_{1}, B_{1}$ et $B 2$ ont été marqués par un spot au bleu alcyan (HART et PITCHER, 1969) sur une nageoire différente pour chaque secteur. Le devenir des 0 + marqués a été suivi sur chacun des trois 
secteurs lors des inventaires réalisés en fin octobre 1985, mais également à la mi-mars 1985. Un piégeage partiel a été pratiqué dans le sens de la descente, au printemps 1984 (du 17 avril au 31 mai), puis 2 semaines par mois, d'octobre à décembre 1984. Le piégeage n'a pu être poursuivi en début 1985 du fait de la prise en glace puis des crues violentes du Redon. Le piégeage partiel a été pratiqué à l'aide d'un piège (largeur : $1,2 \mathrm{~m}$; longueur: $1,5 \mathrm{~m}$; ouverture : $15 \mathrm{~cm}$; maillage: $1 \mathrm{~cm}$ ) prolongé par un guide de $10 \mathrm{~m}$ (maillage : $1 \mathrm{~cm}$ ) permettant de barrer la rivière sur les $4 / 5^{\mathrm{e}}$ de sa largeur.

\subsection{Tests statistiques}

Les données ont été traitées à l'aide de tests statistiques classiques : chi carrè, test $t$, analyse de la variance.

\section{RÉsultats}

\subsection{Les caractéristiques des géniteurs sédentaires}

\subsubsection{Densité et structure d'âge}

La densité totale de truite varie de 20 à 69 ind. $/ 100 \mathrm{~m}^{2}$ selon le secteur et l'année. C'est le secteur aval qui présente les plus faibles densités ( $<35$ ind. $/ 100 \mathrm{~m}^{2}$ ) quelle que soit l'année (Fig. 2). Les truites d'âge $>1$. sont très peu abondantes $\left(0,3\right.$ à 1,6 ind. $\left./ 100 \mathrm{~m}^{2}\right)$ sur les secteurs $A_{1}$ et $B$ alors que leur densité varie entre 6 et 9 ind. $/ 100 \mathrm{~m}^{2}$ sur $\mathrm{B}_{2}$.

1984
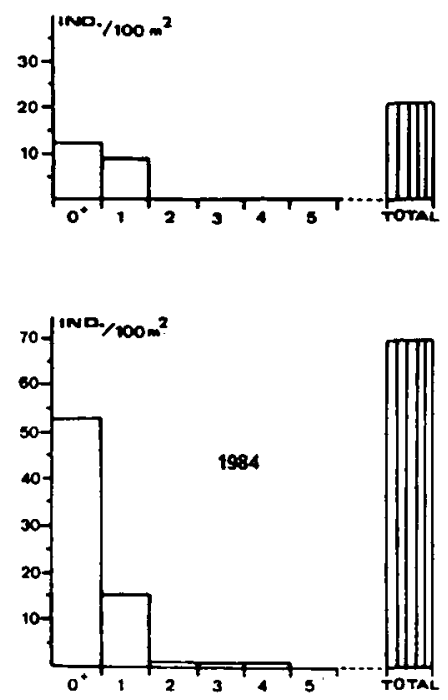

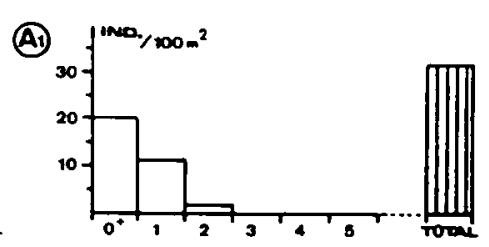

(3)

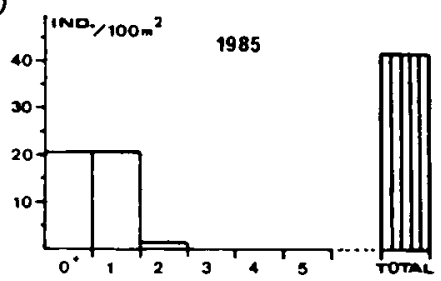

()
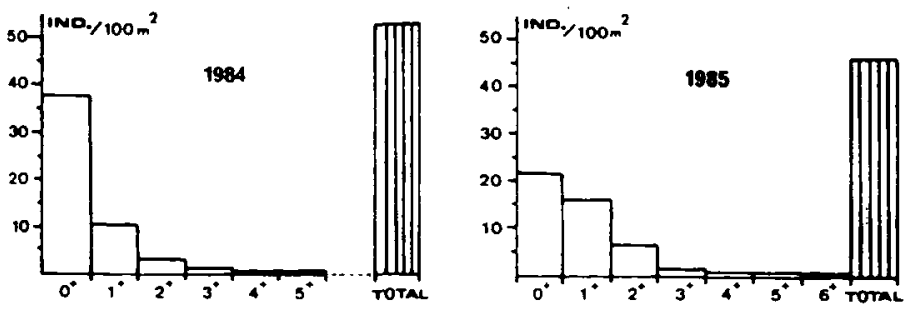

Figure 2 : Densités $\left(\mathrm{N} / 100 \mathrm{~m}^{2}\right)$ de truites sédentaires sur les différents secteurs en fin octobre 1984 et 1985 (ㅁ) densité par classe d’ãge; (⿴囗⿰丿㇄⿱⿴囗⿰丨丨大 $)$ densité totale.

Figure 2 : Density $\left(\mathrm{N} / 100 \mathrm{~m}^{2}\right)$ of sedentary trouts in different sections at the end of october 1984 and 1985 ( $\square$ ) density for different year classes ; (U) total density. 
La maturité est observée dès l'âge 1 - chez les deux sexes (Fig. 3). Dans la classe d'âge 1+il y a selon l'année, de 16 à $27 \%$ de mâles matures contre seulement 3 à $4 \%$ de femelles matures (Tableau 3). Ce n'est qu'à partir du stade 2 + que la quasi totalité des truites sont matures. Les mâles matures représentent selon l'année 25 à $54 \%$ des 2 - contre 41 à $69 \%$ dans le cas des femelles. Les truites d'âge $\geqslant 3$ - sont essentiellement des femelles (Fig. 3 et Tableau III).
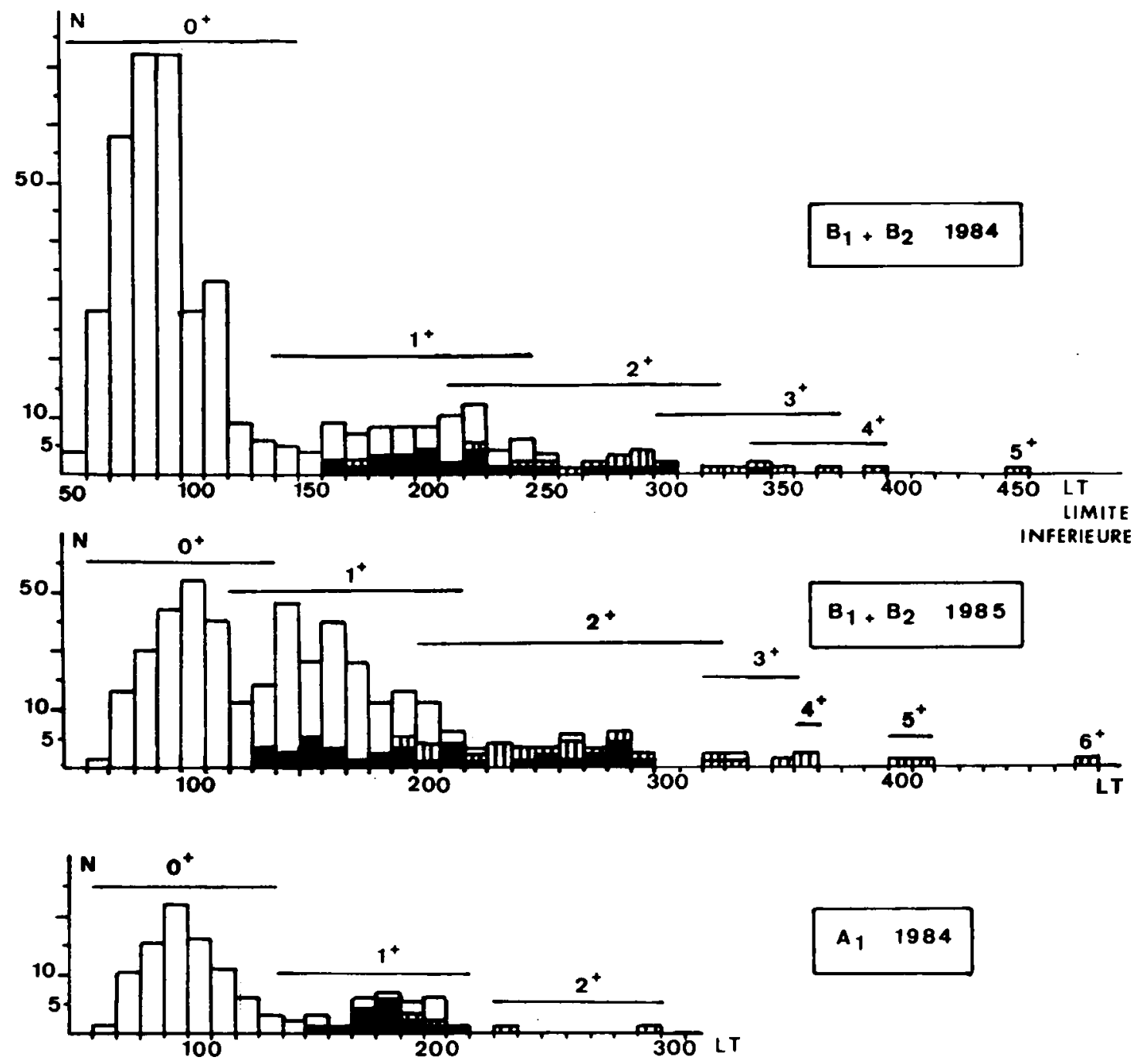

$$
A_{1} 1984
$$

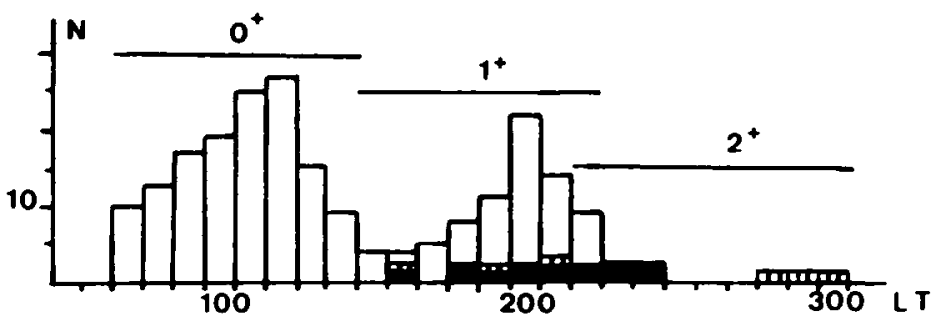

$$
\text { A } 1985
$$

Figure 3 : Truites sédentaires pêchées en fin octobre 1984 et 85 sur la zone $A$ (secteur $A_{1}$ ) et sur

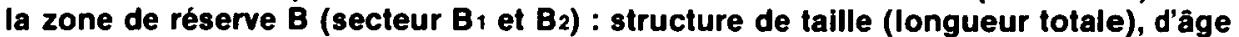
et sexage des géniteurs ((D) mâles spermiant; (W) femelle en vittellogénèse : ( $\square$ ) immature ou mâle non spermiant).

Figure 3 : Sedentary trouts caught by electrofishing at the end of october 1984 and 85 in the zone $A$ (section $A_{1}$ ) and $B$ (sections $B_{1}$ and $\left.B_{2}\right)$ : size structure (total length), age structure and sex-determination of spawners ((E) ripe males; (II) female undergoing vittellogenesis; (ㅁ) immature trout or unripe male). 
Tableau III : Structure démographique (âge et sexe) des truites sédentaires capturées dans le Redon en octobre 1984 et 1985 (secteurs $A_{1}, B_{1}$ et B2 réunis).

Table III : Demographic structure (age and sex) of sedentary trouts caught in River Redon in october 1984 and 1985 (section $A_{1}, B_{1}$ and $B_{2}$ taken together).

\begin{tabular}{|c|c|c|c|c|c|c|c|}
\hline & & & & & & & $\geq 3^{*}$ \\
\hline & & N & $:$ & N & \% & $N$ & $\because$ \\
\hline & $\delta$ sperniant & 40 & 27,4 & 4 & 25,0 & 2 & 25,0 \\
\hline 1984 & \& mature & 5 & 3,4 & 11 & 68,8 & 6 & 75,0 \\
\hline & $\begin{array}{l}\text { Imature ou } \sigma^{7} \\
\text { non speraiant }\end{array}$ & 101 & 69,2 & 1 & 6,2 & 0 & 0 \\
\hline & Total & 146 & 100 & 16 & 100 & 8 & 100 \\
\hline & $\sigma$ spermiant & 32 & 15,8 & 21 & 53,9 & 0 & 0 \\
\hline 1985 & $f$ mature & 8 & 3,9 & 16 & 41,0 & 8 & 88,9 \\
\hline & $\begin{array}{l}\text { Imadure ou } \sigma \\
\text { non sperniant }\end{array}$ & 163 & 80,3 & 2 & 5,1 & 1 & 11,1 \\
\hline & Total & 203 & 100 & 39 & 100 & 9 & 100 \\
\hline
\end{tabular}

La densité en géniteurs mâles varie entre 2,2 et 2,6 ind./100 $\mathrm{m}^{2}$ dans le secteur aval $\mathrm{A}_{1}$ alors qu'elle est plus élevée $\left(3,5\right.$ à 5,8 ind. $\left./ 100 \mathrm{~m}^{2}\right)$ sur les secteurs de la réserve. La densité en femelles mûres varie entre 0,4 et 1 ind. $/ 100 \mathrm{~m}^{2}$ sur les deux secteurs $A_{1}$ et $B_{1}$ alors qu'elle est nettement plus élevée $\left(4,3\right.$ à 6,4 ind. $\left./ 100 \mathrm{~m}^{2}\right)$ sur le secteur $B_{2}$.

\subsubsection{Rapport des sexes}

Sur les secteurs $A_{1}$ et $B_{1}$, le rapport des sexes est nettement en faveur des mâles (Tableau IV) et ne varie pas significativement selon l'année et le secteur. Par contre le sexe-ratio est équilibré sur le secteur B2. II varie peu entre 1984 et 1985 malgré une augmentation globale du nombre de géniteurs qui n'a pas été observée sur les deux autres secteurs (Tableau IV).

\subsubsection{Taille moyenne}

Sur les secteurs de réserve, en liaison avec la structure d'âge (Fig. 3 et Tableau III), la taille moyenne des femelles est toujours supérieure à celle des mâles (Tableau IV). Pour les deux années, la taille moyenne des mâles sur le secteur $B_{2}$ est plus élevée que celle observée sur les deux autres secteurs (Tableau IV). La taille moyenne des géniteurs (2 sexes regroupés) pour les différentes classes d'âge est précisée dans le Tableau Va.

\subsection{Les caractéristiques des captures de géniteurs d'origine lacustre}

Lors des reproductions de 1983, 84 et 85 , respectivement 40,50 et 54 géniteurs de truite de lac ont été capturés dans le Redon.

\subsubsection{Iocalisation et période de présence}

Au cours des trois années, des captures de géniteurs d'origine lacustre ont été réalisées uniquement dans la zone aval A confirmant l'extrême difficulté du passage en amont de la zone A. Plus de $90 \%$ des captures ont été effectuées entre le 10 novembre et le 10 janvier (Fig. 4). Les pics de captures ont été observés pendant et juste après les plus fortes crues (niveaux $>40$ ) (Fig. 4 a et b). Les captures ont été plus tardives en 1983 et 1985 qu'en 1984. En effet, pour cette dernière année, $90 \%$ des captures ont été réalisées avant le 20 décembre alors que ce même pourcentage n'a été atteint que vers le 5 janvier lors des deux autres saisons de reproduction (Fig. 4 b). Le temps de séjour des géniteurs dans le Redon apparaît plus court pour les femelles que pour les mâles : $19 \%$ d'entre elles ont été capturées deux fois contre $33 \%$ dans le cas des mâles. Seuls quelques individus sont encore présents en début mars, à l'ouverture de la pêche. 
Tableau IV : Rapport des sexes $(\circlearrowleft / q)$ et taille moyenne (LF = longueur fourche en mm; $v$ écart type des LF) des géniteurs de truites sédentaires capturés dans le Redon en octobre 1984 et 1985.

Table IV : Sex-ratio $(\delta / q)$ and average length (fork length, LF in $\mathrm{mm} ; \sigma:$ standard deviation of the lengths) of sedentary spawners caught in River Redon in october 1984 and 1985.

\begin{tabular}{|c|c|c|c|c|c|}
\hline iecteur & Année & $\hat{\delta} / q$ & $\because q$ & $\operatorname{Lf}_{m i}^{f}(0)$ & $\operatorname{LF}_{\mathrm{mm}} \delta(0)$ \\
\hline$A_{1}$ & 1984 & $18 / 3$ & 14,3 & $232(56)$ & $185(15)$ \\
\hline$A_{1}$ & 1985 & $16 / 4$ & 20,0 & $210 \quad(45)$ & $217(35)$ \\
\hline$B_{1}$ & 1984 & $14 / 2$ & 12,5 & $310(35)$ & $199(35)$ \\
\hline B. & 1985 & $15 / 3$ & 16,7 & $203(16)$ & $180(38)$ \\
\hline$B_{2}$ & 1994 & $14 / 17$ & 54,8 & $303(67)$ & $247(41)$ \\
\hline$B_{2}$ & 1985 & $22 / 25$ & 53,2 & $291(70)$ & $220 \quad(53)$ \\
\hline$i_{1}+B_{2}$ & 1984 & $20 / 19$ & 40,4 & $304(64)$ & $221(44)$ \\
\hline$B_{1}+B_{2}$ & 1985 & $37 / 29$ & 43,1 & $282(72)$ & $204(51)$ \\
\hline
\end{tabular}

\subsubsection{Rapport des sexes}

Le rapport des sexes ( $̋ / \&$ ) augmente de 1983 à 1985 passant de 1 en 1983 à 1,4 en 1984 et à 2,0 en 1985. Alors que le nombre de femelles capturées est relativement stable, le nombre de mâles passe de 20 à 30 et 36 de 1983 à 1985 (Fig. 4b).

\subsubsection{Structure d'âge (Fig. 5)}

L'âge a pu être déterminé par scalimétrie pour $92 \%$ des captures. Il varie de $2(1+)$ à 6 ans $(5+)$. Les individus de 2 et 3 ans représentent $80,5 \%$ des mâles alors qu'ils ne représentent que $38,2 \%$ dans le cas des femelles. De 1983 à 1985, alors que le nombre de mâles de 3 ans reste relativement stable, le nombre de mâles de 2 ans augmente fortement passant de 2 à 13 puis 26 (Fig. 5). Quelle que soit l'année, le nombre de géniteurs femelles âgés de 4 à 6 ans est toujours supérieur à celui des mâles du même âge.

\subsubsection{Taille (Fig. 6, Tableau Vb)}

La taille des géniteurs de truites capturés varie entre 27 et $82 \mathrm{~cm}$. La structure de taille des mâles est moins étalée que celle des femelles et présente des variations annuelles importantes. En effet, en 1983, $86 \%$ des mâles ont une longueur comprise entre 37 et $48 \mathrm{~cm}$. En 1984 et 1985 , on constate une forte présence de petits mâles: les individus de taille comprise entre 28 et $39 \mathrm{~cm}$ représentent respectivement 63 et $83 \%$ des mâles capturés. La taille moyenne des géniteurs capturés est respectivement de $419 \mathrm{~mm}$ pour les mâles et de $526 \mathrm{~mm}$ pour les femelles. Les tailles moyennes en fonction des différentes classes d'âge sont indiquées dans le Tableau Vb. La comparaison des figures 3 et 6 fait apparaitre nettement, pour les géniteurs de truite de lac la présence de grands individus qui n'ont jamais été trouvés dans les inventaires de truites sédentaires dans lazone $A(L T<30 \mathrm{~cm})$ et $B(L T<50 \mathrm{~cm})$. Pour chacune des classes d'âge $1+$ à $5+$, la taille moyenne des géniteurs lacustres est très nettement supérieure à celle des truites sédentaires (Tableaux Va et Vb). Les différences sont statistiquement très significatives $(\alpha<1 \% 0)$ pour les classes d'âge $1+$ et $2+$. 

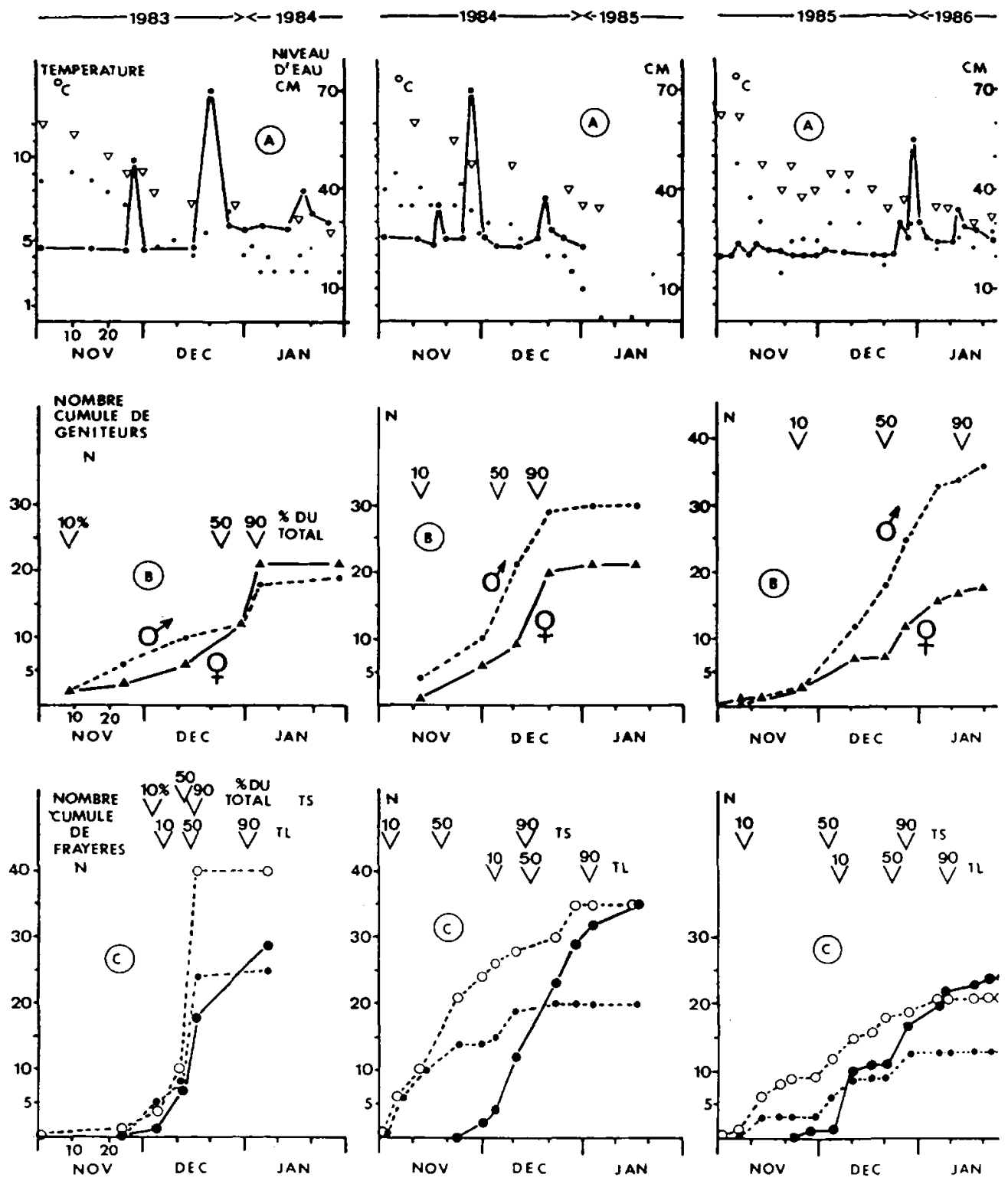

Figure 4 : SUIVI DE LA FRAIE DE LA TRUITE SUR LE REDON AU COURS DE TROIS SAISONS DE REPRODUCTION: 1983-84; 1984-85; 1985-86.

Figure 4a : Paramètres physiques $($. ) température du Redon; $(\nabla)$ temperature du lac en bordure; (._..) niveau d'eau du Redon.

Figure 4b : Évolution du nombre cumulé de géniteurs de truites de lac capturées: (•-_.) mâles ; $(\boldsymbol{\Delta}-\boldsymbol{\Delta})$ femelles.

Figure 4c : Évolution du nombre cumulé de frayères de truites comptées sur les zones $A$ et $B$ du Redon. Frayères de truites sédentaires (T.S.) sur la zone A (._...) et sur la zone $B$ $(O-O)$. Frayères de trultes de lac (T.L.) sur la zone $A(\longrightarrow)$.

FIgure 4 : SURVEY OF THE REPRODUCTION OF TROUT ON THE RIVER REDON DURING THREE SPAWNING PERIODS : 1983-84; 1984-85; 1985-86.

Figure 4a : Physical parameters: (.) temperature of the Redon; $(\nabla)$ temperature of Lake Leman near the shore; (. - ) water level of the River Redon.

Figure $4 b$ : Evolution of the cumulative number of trouts from the lake caught in the River Redon: (.--..) males; $(\Delta-\Delta)$ females.

Figure 4c : Evolution of the cumulative number of trout redds counted in the section $A$ and $B$ of the River Redon. Redds of sedentary trouts (T.S.) in section $A$ (..-..) and in section B (O--O) . Redds of trouts from the Lake Leman (T.L.) in the section A (. $\multimap$ ). 


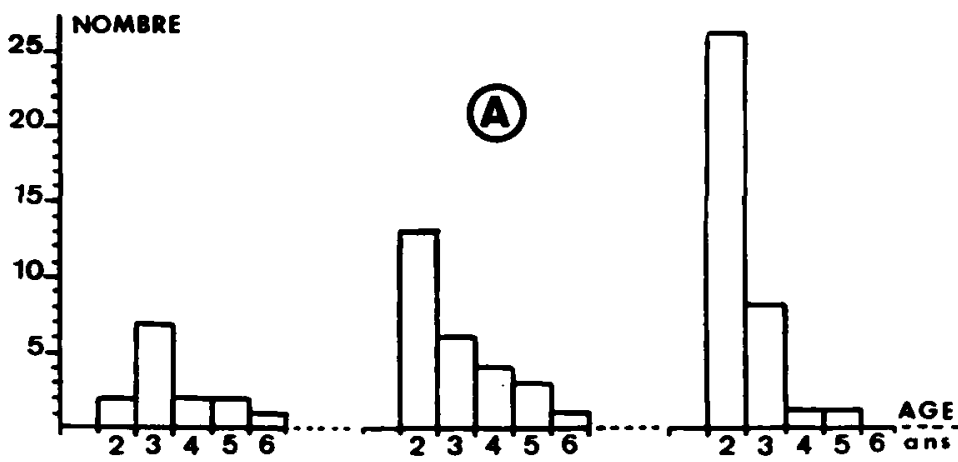

(B)
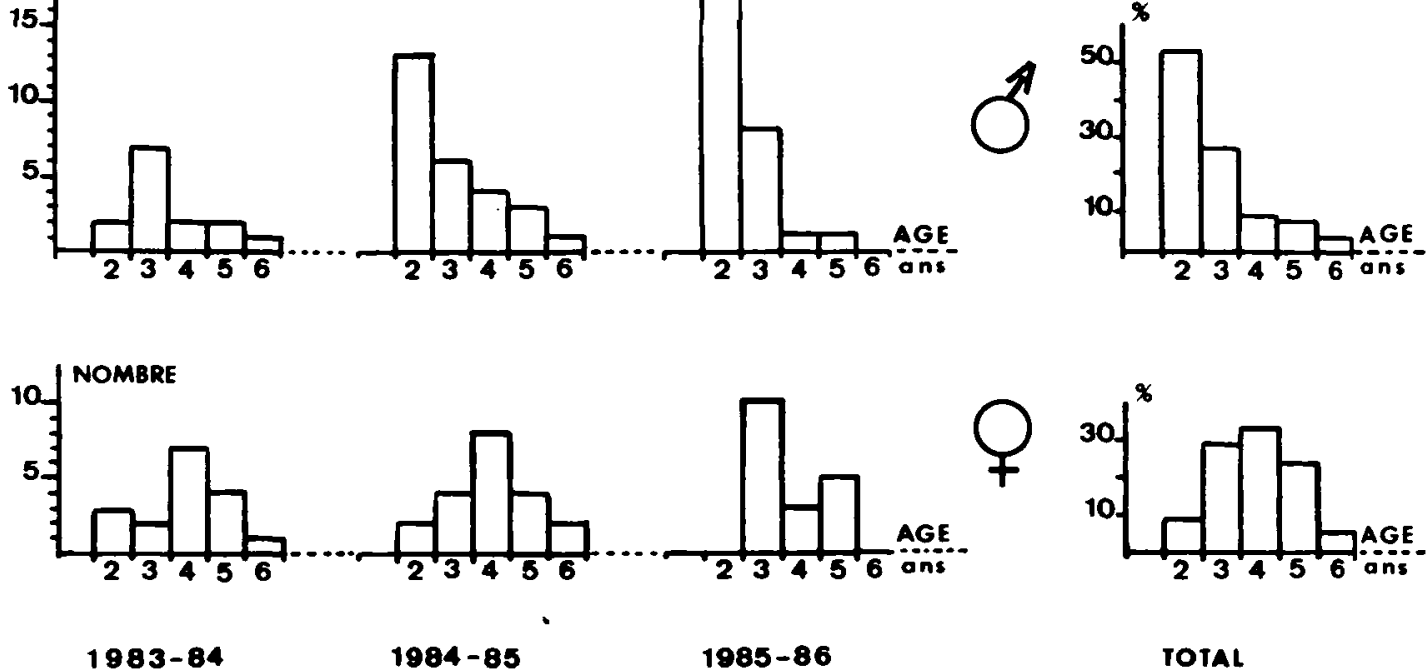

$1883-84$

$1984-85$

$1985-86$

TOTAL

Figure 5 : STRUCTURE D'AGE DES GENITEURS DE TRUITE DE LAC CAPTURES DANS LE REDON ET DONT L'AGE A ÉTÉ DÉTERMINÉ (92\% DES CAPTURES). (2) $1+$; (3) 2 +; (4) $3+$; (5) $4+$; (6) $5+$.

Figure 5a : Effectifs par classe d'åge et par sexe pour chacune des 3 saisons de reproduction.

Figure 5b : Pourcentage des différentes classes d'åge pour chacun des sexes sur l'ensemble des truites capturées et dont l'âge a été déterminé.

Figure 5 : AGE STRUCTURE OF LAKE DWELLING TROUT SPAWNERS CAUGHT IN THE RIVER REDON AND AGED (92 \% OF CATCHES). (2) $1+$; (3) $2+$; (4) $3+;$ (5) $4+;$ (6) $5+$.

Figure 5a : Number per year-class and per sex for the three different seasons of reproduction.

Figure 5b : Percentage of the different year classes for each sex for the total of trouts caught and aged.

\section{3. la fraie}

\subsubsection{Répartition spatiale (Tableau VI)}

La truite de lac a frayé exclusivement dans la partie aval $A$ du Redon où la densité moyenne estimée des frayères a varié entre 3,8 et $4,5 / 1.000 \mathrm{~m}^{2}$ selon l'année. La truite sédentaire se reproduit dans les affluents et sur l'ensemble du cours principal. Sur ce dernier la densité moyenne de frayères varie de 2 à $16,7 / 1.000 \mathrm{~m}^{2}$. Quelle que soit l'année, elle est nettement supérieure (4,4 à 4,8 fois) sur la zone $B$ (réserve de pêche) que sur la zone aval $A$. Sur chacune des deux zones, le nombre de frayères de truites sédentaires diminue de 1983 à 1985.

\subsubsection{Déroulement dans le temps (Fig. 4)}

Chaque année, la fraie est en moyenne plus tardive pour la truite de lac que pour la truite sédentaire. En effet, lorsque $50 \%$ des frayères de truite de lac sont creusées, le pourcentage de celles de truite sédentaire est déjà voisin de $90 \%$ (Fig. 4C).

Pour la truite sédentaire, l'activité maximale de fraie se situe sensiblement à la même période dans les zones $A$ et B. Elle a lieu entre le début novembre et la mi-décembre en 1984 et la fin décembre en 1985. En 1983, l'activité maximale de fraie s'est déroulée sur une période très courte (première quinzaine de décembre). Chez la truite de lac, l'activité maximale de fraie a lieu entre le début décembre et le début janvier quelle que soit l'année. 
Tableau $V$ : TAILLE MOYENNE (LT : LONGUEUR TOTALE EN mm; $n$ : NOMBRE ; $\sigma$ : ECART TYPE DES LONGUEURS) PAR CLASSE D'AGE.

Table $V$ : MEAN TOTAL LENGTH (LT : LONGUEUR TOTALE IN mm;n:NUMBER; $\sigma$ : STANDARD DEVIATION OF THE LENGTHS) FOR DIFFERENT AGE-CLASSES.

Tableau Va : Ensemble des géniteurs de truite sédentaire sexés en fin octobre 1984 et 1985 dans le Redon.

Table Va : Sedentary trout spawners controlled in the River Redon at the end of october 1984 and 1985.

\begin{tabular}{|c|c|c|c|c|c|}
\hline $\begin{array}{c}\text { Classe d‘ àge } \\
\text { (Age) }\end{array}$ & $\begin{array}{c}1^{+} \\
(2)\end{array}$ & $\begin{array}{c}2^{+} \\
(3)\end{array}$ & $\begin{array}{c}3^{+} \\
(4)\end{array}$ & $\begin{array}{c}4^{+} \\
(5)\end{array}$ & $\begin{array}{c}5^{+} \\
(6)\end{array}$ \\
\hline $\begin{array}{c}\text { LT J+f } \\
\left(n ; 0^{+}\right.\end{array}$ & $\begin{array}{c}191 \\
(83 ; 24)\end{array}$ & $\begin{array}{c}268 \\
(53 ; 25)\end{array}$ & $\begin{array}{c}341 \\
(8 ; 23)\end{array}$ & $\begin{array}{c}365 \\
(4 ; 27)\end{array}$ & $\begin{array}{c}421 \\
(3 ; 26)\end{array}$ \\
\hline
\end{tabular}

Tableau Vb : Ensemble de géniteurs de truite de lac capturés dans le Redon (de l'hiver 1983-84 à 1985-86) et dont l'âge a été déterminé par scalimétrie.

Table Vb : Lake dwelling trout spawners caught in the River Redon during three spawning runs (1983-84 to 1985-86) and aged by scale-reading.

\begin{tabular}{|c|c|c|c|c|c|}
\hline $\begin{array}{c}\text { Classe d' àge } \\
\text { (Age) }\end{array}$ & $\begin{array}{l}1^{+} \\
(2)\end{array}$ & $\begin{array}{l}2^{+} \\
\text {(3) }\end{array}$ & $\begin{array}{l}3^{+} \\
(4)\end{array}$ & $\begin{array}{r}4^{+} \\
(5)\end{array}$ & $\begin{array}{r}5^{+} \\
(6)\end{array}$ \\
\hline $\begin{array}{l}\left.\sigma^{\pi} \quad L T ; 0\right) \\
\quad(n ; 0)\end{array}$ & $\begin{array}{c}343 \\
(41 ; 31)\end{array}$ & $\begin{array}{l}397 \\
(21 ; 50)\end{array}$ & $\begin{array}{l}517 \\
(7 ; 99)\end{array}$ & $\begin{array}{c}700 \\
(6 ; 52)\end{array}$ & $\begin{array}{c}743 \\
(2 ; 67)\end{array}$ \\
\hline $\begin{array}{c}f \quad \text { LT } \\
(n ; 0)\end{array}$ & $\begin{array}{c}362 \\
(5 ; 32)\end{array}$ & $\begin{array}{c}371 \\
(16 ; 56)\end{array}$ & $\begin{array}{c}559 \\
(18 ; 75)\end{array}$ & $\begin{array}{c}691 \\
(13 ; 88)\end{array}$ & $\begin{array}{c}763 \\
(3 ; 52)\end{array}$ \\
\hline $\begin{array}{l}\text { TOTAL LT } \\
(n ; 0)\end{array}$ & $\begin{array}{c}343 \\
(46 ; 31)\end{array}$ & $\begin{array}{l}385 \\
(37 ; 53)\end{array}$ & $\begin{array}{c}547 \\
(25 ; 85)\end{array}$ & ${ }^{694}(19 ; 79)$ & $\begin{array}{c}755 \\
(5 ; 59)\end{array}$ \\
\hline
\end{tabular}

L'activité de fraie des deux populations de géniteurs peut débuter avant l'arrivée des crues. Au cours de l'hiver 1985-86 la totalité de la fraie de la truite sédentaire s'est déroulée en période de basses eaux (Fig. $4 \mathrm{a}$ et $\mathrm{C}$ ).

Au cours des périodes d'activité maximale de fraie, la température du Redon, toujours inférieure à celle du lac, a varié entre 8 et $2^{\circ} \mathrm{C}$ (Fig. 4).

\subsection{Caractéristiques des juvéniles $\mathrm{O}_{+}$et $1+$ et leur devenir}

\subsubsection{Densité (Fig. 2)}

Les densités automnales en 0 + varient entre 12 et 53 ind. $/ 100 \mathrm{~m}^{2}$ selon l'année et le secteur. En 1984 , la densité est nettement plus faible $\left(12 \mathrm{ind} . / 100 \mathrm{~m}^{2}\right)$ sur le secteur aval $\mathrm{A}_{1}$ comparativement à celles observées sur les deux autres secteurs $\left(38\right.$ à 53 ind. $\left./ 100 \mathrm{~m}^{2}\right)$. En 1985, la densité en 0 + est voisine de 20 ind. $/ 100 \mathrm{~m}^{2}$ sur les trois secteurs. La densité automnale en 1 + varie entre 8 et 21 ind. $/ 100 \mathrm{~m}^{2}$ et, quelle que soit l'année, la densité est supérieure sur $\mathrm{B}_{1}$, comparativement aux deux autres secteurs. 

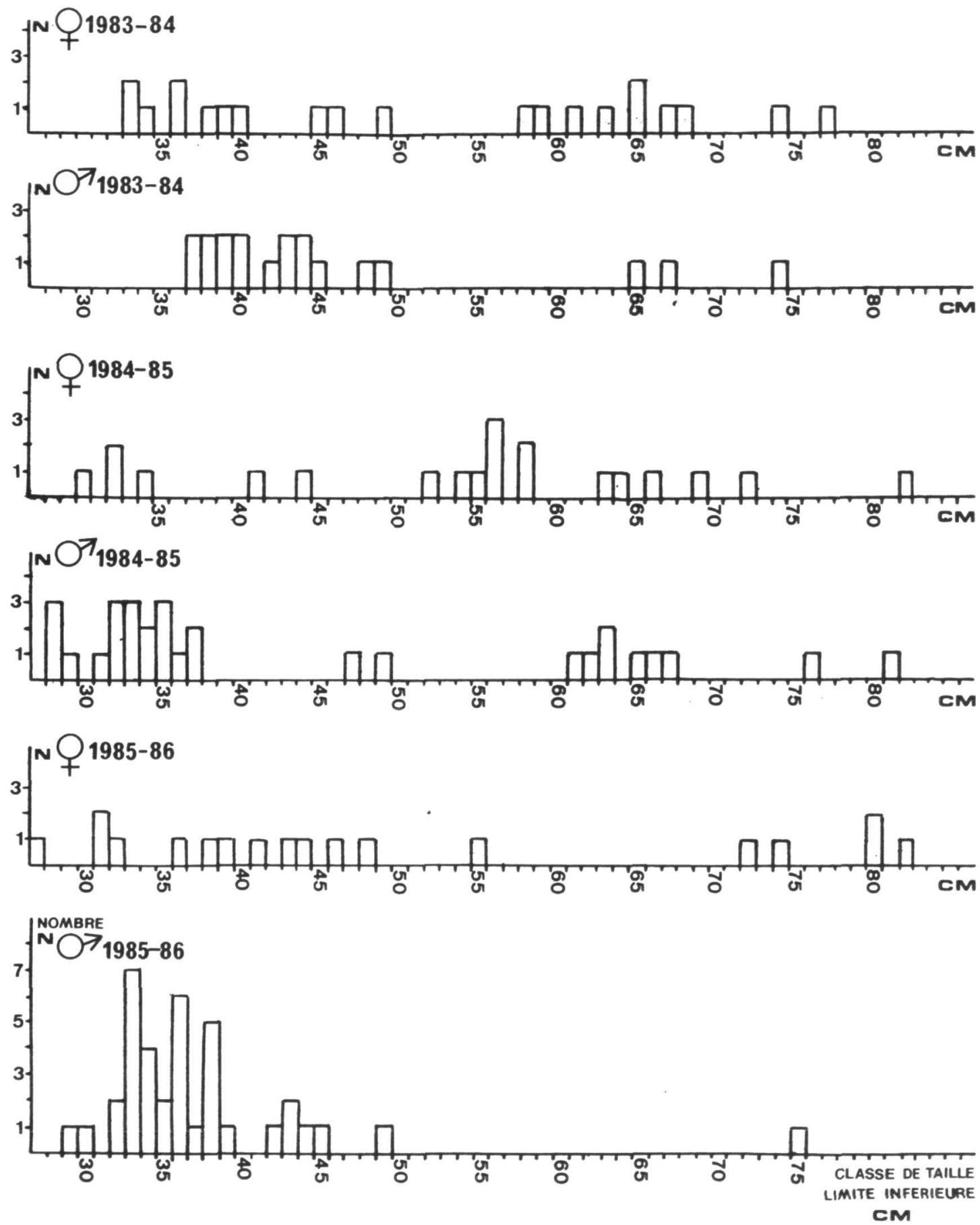

Figure 6 : Structure de taille (longueur totale) des géniteurs de trulte de lac capturés dans le Redon de 1983-84 à 1985-86.

Figure 6 : Size structure (total length) of lake dwelling trout spawners caught in the River Redon during the winter 1983-84 to 1985-86.

\subsubsection{Taille moyenne (Fig. 7)}

la taille moyenne des 0 + et 1 + varie respectivement de 85 à $105 \mathrm{~mm}$ et de 159 à $213 \mathrm{~mm}$ selon l'année et le secteur. La taille des $0+$ en 1984 et 1985 est significativement $(\alpha \leq 5 \%)$ inférieure sur le secteur $B_{1}$, comparativement à celle observée pour la même année sur les deux autres secteurs. Par ailleurs, pour chacun des secteurs, la taille moyenne des $0+$ est significativement $(\alpha<5 \%)$ plus élevée en 1985 qu'en 1984. 


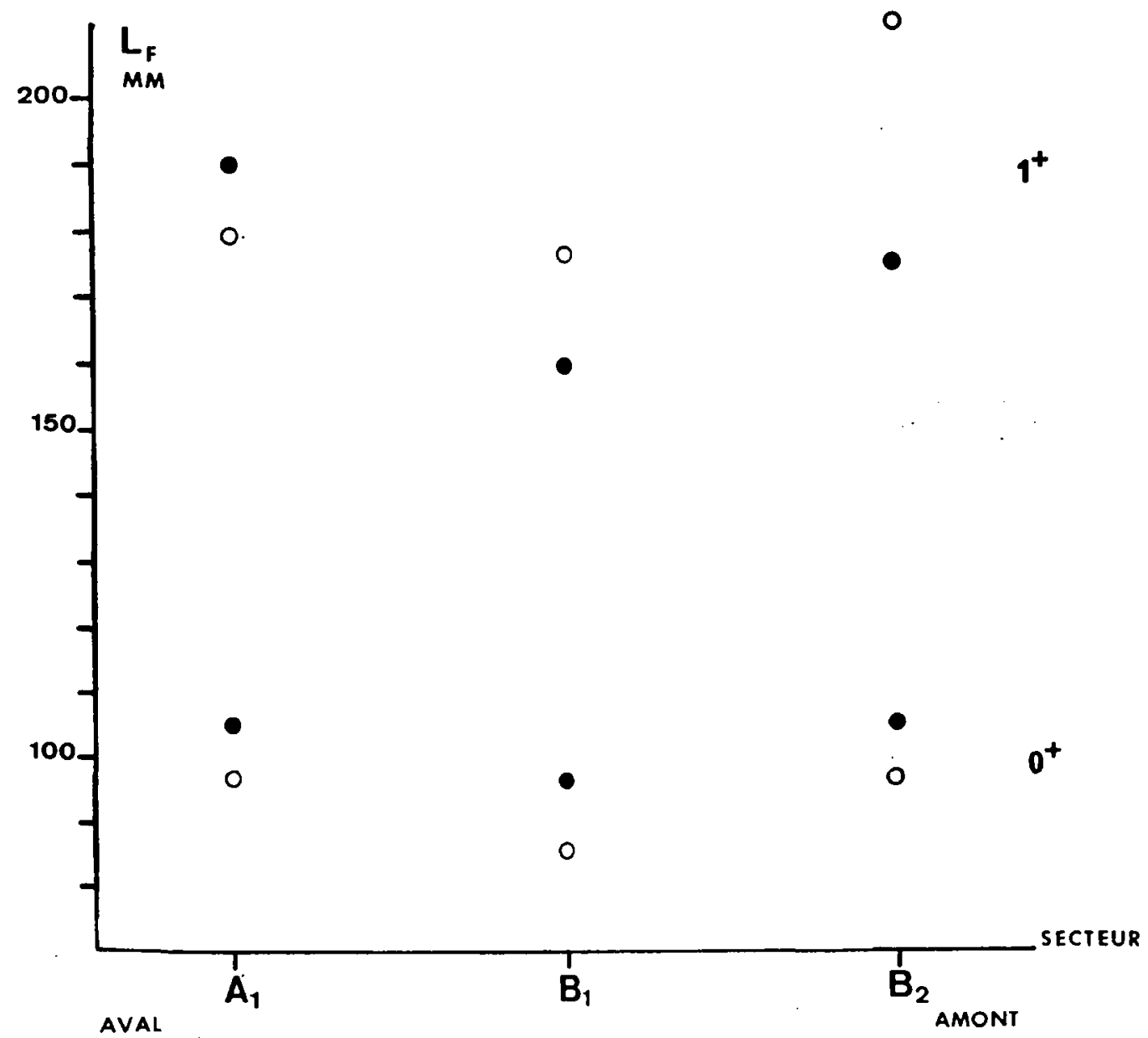

Figure 7 : Taille moyenne (LF en $\mathrm{mm}$ ) des juvéniles $0_{+}$et 1 + de truites capturés dans les secteurs $A_{1}, B_{1}$ et $B_{2}$ du Redon en octobre 1984 et 1985. (o) : 1984; (•) : 1985.

Figure 7 : Average length (LF in $\mathrm{mm}$ ) of resident juveniles caught in section $A_{1}, B_{1}$ and $B_{2}$ of River Redon in october 1984 and 1985. (०) : $1984 ;(\bullet): 1985$.

Tableau VI : Densité (nombre/1000 $\mathrm{m}^{2}$ ) des frayères de truites de lac et de truites sédentaires dans les zones A et B du Redon de 1983-84 à 1985-86.

Table VI : Density (number/1000 $\mathrm{m}^{2}$ ) of redds of lake dwelling trouts and sedentary trouts in zones A and B of River Redon from 1983-84 to 1985-86.

\begin{tabular}{|l|c|c|c|c|}
\hline $\begin{array}{l}\text { Densité de } \\
\text { frayères } \\
\text { Nonbre } 10000^{2}\end{array}$ & \multicolumn{2}{|c|}{ Truite de lac } & \multicolumn{2}{c|}{ Truite sédentaire } \\
\hline $\begin{array}{l}\text { Hiver de } \\
\text { reproduction }\end{array}$ & Zone A & Zone B & Zone A & Zone B \\
\hline $1983-84$ & 4,5 & 0 & 3,9 & 16,7 \\
\hline $1984-85$ & 5,5 & 0 & 3,1 & 14,8 \\
\hline $1985-86$ & 3,8 & 0 & 2,0 & 8,8 \\
\hline
\end{tabular}




\subsubsection{Rapport des sexes}

Dans un échantillon de 58 truitelles $0+$ de la zone $A$ sexées après autopsie en 1985, le rapport des sexes est de $35 \hat{\delta}^{\prime} / 23 \%$. II n'y a pas de différence significative $(\alpha<5 \%)$ entre la taille moyenne des mâles $(94,5 \mathrm{~mm})$ et celle des femelles $(96,5 \mathrm{~mm})$.

\subsubsection{Devenir des juvéniles $0_{+}$}

Recapture des truitelles $0_{+}$marquées en octobre 1984 (Tableau VII)

Le taux de recapture en $1+$ (octobre 1985) est faible (13 a $23 \%$ ) et ne varie pas significativement selon les secteurs. Au stade 1+, la part des individus marqués sur le même secteur en $0+$ est faible et augmente de l'aval vers l'amont ( 25 à $40 \%$ ) mais les différences ne sont pas significatives.

Dès mars 1984 (fin du stade $0_{+}$) les juvéniles marqués nè représentaient plus que 29 à $56 \%$ des 0 + dans leur secteur d'origine. Le taux de recapture était déjà très faible $(15$ à $28 \%)$ et il a peu varié entre mars 1984 et octobre 1985.

Deux truitelles $0+$ du secteur amont $B_{2}$ ont été retrouvées dans des secteurs aval : une en $B_{1}$ en mars 1985 et l'autre en $A_{1}$ en octobre 1985.

Évolution de la densité

Dans les secteurs $B_{1}$ et $B_{2}$ on observe une forte chute (voisine de $50 \%$ ) de la densité $0_{+}$entre la fin octobre 1984 et la mi-mars 1985 . Le phénomène est moins marqué dans le cas du secteur aval $A_{1}$.

Dévalaison vers le lac

Bien que très limitées, les opérations de piégeage partiel ont permis de mettre en évidence l'existence de dévalaisons vers le Léman de truitelles de 1 été. Douze juvéniles 0 +ont été capturés en fin automne 1984; leur taille variait de 65 à $135 \mathrm{~mm}(\bar{L}=92)$. Dix neuf juvéniles de 1 an (début du stade $1+$ ) ont été capturés en fin avril et en mai 1984. Leur taille était comprise entre 123 et $173 \mathrm{~mm}$ $(\bar{L}=151)$. Au cours de cette même période, deux truites de 2 ans (début du stade $2+$ ) ont également été piégées. Les truitelles capturées dévalant au printemps présentaient un aspect argenté et un coefficient $(K=1,03)$ faiblement mais significativement $(\bar{\alpha}<5 \%)$ plus faible que celui $(k=1,14)$ des truitelles en place.

Tableau VII : Devenir des 0 , marqués en octobre 1984 ; présence en fin 0 + (mars 1985) et en 1 - (octobre 1985).

Table VII : Recapture (in march and october, 1985) of trouts marked at 0 -stage in october 1984.

\begin{tabular}{|c|c|c|c|c|c|c|c|c|}
\hline \multirow{3}{*}{\begin{tabular}{|l}
$0+\begin{array}{l}\text { (octobre } \\
\text { 1984) }\end{array}$ \\
$\begin{array}{l}\text { Secteur } \\
\text { (nonbre de } \\
\text { arqués) }\end{array}$
\end{tabular}} & \multicolumn{4}{|c|}{ fin o+ (ars 1985) } & \multicolumn{4}{|c|}{ 1+ (fin octobre 1985) } \\
\hline & \multirow{2}{*}{$\begin{array}{l}\text { : de } \\
\text { marqués } \\
\text { retrouvés }\end{array}$} & \multicolumn{3}{|c|}{ Nonbre de arqués } & \multirow{2}{*}{$\begin{array}{c}\text { de } \\
\text { marqués } \\
\text { retroweses }\end{array}$} & \multicolumn{3}{|c|}{ Mombre de marqués } \\
\hline & & $A_{1}$ & $B_{1}$ & $B_{2}$ & & $A_{1}$ & $B_{1}$ & $B_{2}$ \\
\hline$A_{1} \quad(n=84)$ & 20,2 & 17 & 0 & 0 & 22,6 & 19 & 0 & 0 \\
\hline$B_{1} \quad(n=161)$ & 28,0 & 0 & 45 & 0 & 13,0 & 0 & 21 & 0 \\
\hline \multirow{3}{*}{$\begin{array}{l}B_{2} \quad(n=151) \\
z=z=-z=:=\end{array}$} & 14,6 & 0 & 1 & 22 & $16,6$. & 1 & 0 & 25 \\
\hline & $\begin{array}{l}=: z=z z=z= \\
\text { Nbre de } 0^{*} \\
\text { dans le } \\
\text { secteur }\end{array}$ & $\begin{array}{r}z=-2= \\
59\end{array}$ & $\begin{array}{c}z=z=z \\
80\end{array}$ & $\begin{array}{c}z=z=z= \\
75\end{array}$ & $\begin{array}{l}=:=z=z=z= \\
\text { Nre de 1* } \\
\text { dans le } \\
\text { secteur }\end{array}$ & $z=z=z=-$ & $\begin{array}{c}n=:= \\
64 \\
64\end{array}$ & 67 \\
\hline & $\begin{array}{l}: \text { de } 0^{+} \\
\text {originaires } \\
\text { du secteur }\end{array}$ & 28,8 & 56,2 & 29,3 & $\begin{array}{l}\text { : de } 1^{+} \\
\text {originaires } \\
\text { du secteur }\end{array}$ & $5.25,0$ & 32,8 & 39,7 \\
\hline
\end{tabular}




\section{DISCUSSION}

\subsection{Géniteurs sédentaires en place avant la fraie}

La technique de sexage utilisée a permis de mettre en évidence, sur le cours principal du Redon, une grande hétérogénéité dans la distribution spatiale des géniteurs sédentaires en place avant la fraie. Dans la zone aval, ouverte à la pêche et fréquentée par les géniteurs de truite de lac, le rapport des sexes est très en faveur des mâles. Le déséquilibre réel pourrait même être plus fort puisque la technique d'identification des mâles par spermiation sous-estime leur nombre. La faible taille et le faible nombre des femelles sédentaires dans la zone aval du Redon suggèrent l'existence d'un potentiel reproducteur négligeable devant celui des truites migrantes. Par contre la zone de réserve amont se caractérise par une densité nettement plus élevée de truites d'âge $>1$ - et par un rapport des sexes plus équilibré chez les individus matures. Plusieurs facteurs explicatifs peuvent être avancés:

- la zone de réserve possède un habitat plus favorable aux individus de grande taille qui sont en majorité des femelles. MAISSE et al., (1987) ont mis en évidence, dans le cas d'un ruisseau breton, un meilleur taux de survie annuel des femelles comparativement aux mâles.

- la taille légale de capture fixée à $21 \mathrm{~cm}$, longueur totale, protège peu, sur la zone aval, les futures femelles 2 . En effet, en début de saison de pêche la taille moyenne (LT) des truites de 2 ans est déjà comprise entre 18 et $20 \mathrm{~cm}$. La mortalité par pêche semble être un des principaux facteurs explicatifs du faible potentiel reproducteur des truites sédentaires sur la zone $A$.

\subsection{Géniteurs venus du lac}

\section{Activité de remontée}

La période de remontée des géniteurs de truites de lac dans le Redon semble correspondre aux observations réalisées en Europe méridionale soit de novembre à janvier (MELHAOUI, 1985). Notons cependant l'existence de remontées plus précoces (été et début automne) dans la Dranse, un grand affluent du Léman. L'existence de remontées très tardives n'est pas exclue puisque des femelles mùres n'ayant pas encore frayé sont parfois capturées en février dans le Léman. Dans le Redon, les pics de remontées apparaissent principalement liés au facteur "crues", confirmant l'importance de ce paramètre sur les remontées déjà notée par MUNRO et BALMAIN (1956), RUNNSTROM (1957), STUART (1957), THORPE (1974), BAGLINIERE et al., (1987). Ceci pourrait expliquer les remontées tardives observées en 1985, année où les débits sont restés faibles jusqu'à la fin de l'automne. Cependant, selon STUART (1953 et 57) la température semble, dans le cas des trois petits lacs écossais, intervenir sur certaines phases de la migration. Selon l'auteur, les remontées commencent quand la température des affluents devient inférieure à celle du lac et elles sont achevées quand la température des rivières atteint $6-7^{\circ} \mathrm{C}$.

\section{Caractéristiques}

Le non examen des truites de taille inférieure à $25 \mathrm{~cm}$ peut avoir conduit à une sousestimation des petits géniteurs d'âge 1 + et à une surestimation de la taille moyenne des truites de lac $1+$. Néanmoins, pour un âge donné $(>1+)$, les géniteurs de truite de lac ont une taille nettement supérieure à celle des truites sédentaires. Selon MELHAOUI (1985), cette forte croissance serait principalement liée à la vie en milieu lentique et à une alimentation à base de poissons.

Sur 87 géniteurs capturés, marqués et relâchés dans le Redon, lors de la fraie de 1983 et celle de 1984, seulement 3 ont été recapturés dans le Redon lors de la fraie de 1984 et celle de 1985. Ce dernier chiffre apparaît très faible, bien que sans doute sous-estimé du fait du contrôle non total des remontées. Plusieurs raisons peuvent être évoquées:

- la mortalité (naturelle et par pêche). Sur l'ensemble des géniteurs capturés dans le Redon, environ $10 \%$ (surtout les truites âgées de 4 à 6 ans) sont atteints de "syndrome mycosique". Par ailleurs, la mortalité par pêche peut être importante puisque sur 40 géniteurs marqués au cours de l'hiver 1983-84, 13 recaptures par pêche ont été déclarées (MELHAOUI, 1985).

- l'existence d'un phénomène de repos de reproduction. Plusieurs auteurs (ARVIDSON, 1935; ALM, 1949; RUNNSTROM, 1952; STUART, 1953; SAKOWICZ, 1961) signalent qu'une partie des géniteurs de plusieurs lacs peut marquer un arrêt de reproduction (généralement 1 à 2 ans) entre deux fraies. Ce phénomène semble également exister au Léman (MELHAOUI, 1985).

- les phénomènes de divagation. Un géniteur marqué en 1983 dans le Redon a été recapturé en tant que reproducteur l'année suivante dans un affluent de la rive suisse du Léman à $12 \mathrm{~km}$ du Redon. Cependant BUTTIKER et MATTHEY (1986) ont montré, dans le cas de plusieurs affluents suisses du Léman, que les géniteurs à fraies multiples se reproduisent généralement dans le même affluent. 


\subsection{Etudes des frayères et de la traie}

Selon SHIRVELL et DUNGEY (1983), le choix du site de fraie est lié aux caractéristiques du microhabitat: substrat, hauteur d'eau et vitesse du courant. Les valeurs de ces paramètres sont, dans les cas de frayères du Redon (MELHAOUI, 1985), globalement comparables à celles notées par NIHOUARN (1983) et SHIRVELL et DUNGEY (1983). Dans le cas du Redon, les frayères à truites de lac sont généralement plus grandes, plus profondes et avec un substrat plus grossier que celles des truites sédentaires (MELHAOUI, 1985). Les différences sont particulièrement nettes dans la partie aval du Redon ou் les femelles sédentaires sont de petite taille $(<30 \mathrm{~cm})$. Selon OTTAWAY et al. (1981), la taille de la femelle est un facteur majeur influençant la taille des frayères. Il importe cependant d'être très prudent quant à la généralisation des caractéristiques des frayères du Redon à l'ensemble des affluents du Léman. Selon BAGLINIERE et al. (1979), le débit d'eau joue un rôle important dans la répartition spatiale des frayères. Les crues avant la fraie ont un rôle favorable en nettoyant ies sites de fraie et en diminuant la compacité du substrat (STUART, 1953) mais peuvent jouer un rôle néfaste pendant la fraie ou l'incubation (MCNEIL et AHNEIL, 1964 ; CUINAT, 1974). Dans le cas du Redon, en l'absence de crue automnale et donc de nettoyage, les frayères de 1985 ont été réalisées sur des sites à substrats non stabilisés. Ensuite, environ $20 \%$ des frayères ont été soit complétement ensablées soit complètement détruites lors de la première grosse crue de l'hiver 85-86. Des phénomènes de surcreusements ont été observés dans le cas de la truite sédentaire dans la zone de réserve du Redon (MELHAOUI, 1985); ils ont été confirmés par l'ouverture de certaines frayères. Cette observation est un indice de saturation des zones de fraie puisque selon GREELEY (1932), HOBBS (1940), BAGLINIERE et al. (1979), le phénomène est favorisé lorsqu'il y a une surdensité en géniteurs sur les zones de fraie disponibles.

Le comptage des frayères constitue un indice relatif mesurable de l'intensité de fraie sous réserve de la réalisation de plusieurs passages couvrant l'ensemble de la période de fraie. Cette dernière coïncide globalement à celle observée en Bretagne par NIHOUARN (1983) mais se caractérise, dans le cas du Redon, par un léger décalage dans le temps de la fraie de la truite de lac et de la truite sédentaire. Le refroidissement automnal de l'eau, plus rapide en rivière qu'en lac, pourrait être un des facteurs expliquant la reproduction plus précoce des truites sédentaires. Les conséquences de ce décalage restent à étudier d'autant plus que, selon HELAND (1977), la prise de territoire est plus facile pour les alevins précoces. Dans la présente étude, le rapport entre le nombre estimé de frayères de truites de lac et le nombre de femelles capturées a varié selon l'année de 1,3 à 1,7. Cependant il apparaît difficile d'évaluer le nombre de femelles à partir du nombre de frayères. En effet, selon EUZENAT et FOURNEL (1976) et NIHOUARN (1983), certaines femelles de truite peuvent creuser plusieurs frayères. Par ailleurs, d'après BAGLINIERE et al. (1979), le nombre de frayères par femelle peut varier en fonction du milieu ou de l'année.

\subsection{Les juvéniles et leur devenir}

La structure de population de truite du Redon se caractérise par la dominance des classes d'âge 0 . et 1 +qui ont une forte croissance comparativement à d'autres cours d'eau (MELHAOUI, 1985). Les inventaires réalisés ont mis en évidence une forte diminution de la densité en 0 +entre la fin octobre et le début mars. Cette diminution n'est pas attribuable seulement à la mortalité naturelle mais également à des dévalaisons vers le lac, confirmées lors de l'opération de piégeage réalisée en 1984. Le piégeage indique également l'existence de dévalaisons en lac au stade $1+(2$ ans) en fin d'hiver-début de printemps. Ces observations partielles sont confirmées par l'examen des écailles des géniteurs de truite de lac qui indique la présence d'une ou deux années de croissance initiale plus faible "type rivière". L'expérience de marquage réalisée à l'automne 84 et les inventaires de 1985 confirment l'existence de mouvements vers l'aval et indiquent que d'importants brassages aux stades $0+$ et $1+$ ont lieu de la fin automne au début du printemps.

Dans l'écosystème lac-afluents, du fait de l'absence de barrière liée à la salinité, les mouvements des truites entre la zone courante et la zone lentique peuvent prendre des modes plus variés que dans l'écosystème rivière-mer (truite de mer). Dans le cas de la truite de lac (STUART, 1957; THORPE, 1974 ; MELHAOUI, 1985), la dévalaison est plus étalée dans le temps et peut concerner des truites de plus petite taille que celle observée chez les smolts de truite de mer (RICHARD, 1986).

Les campagnes de marquage de truitelles $0+$ observées dans le Léman et dans le Redon (MELHAOUI, 1985; CHAMPIGNEULLE, 1987) indiquent que ces modes de repeuplement peuvent contribuer au recrutement en truitelles et en géniteurs de truite de lac dans le Redon.

\section{CONCLUSION}

La présente étude visait principalement à réaliser une première approche semi-quantitative de la fraie de la truite (lacustre et sédentaire) dans un affluent du Léman. L'étude comparative de l'activité de fraie des truites lacustres et sédentaires a été rendue possible grâce à une bonne connaissance des caractéristiques et de la répartition des deux types de géniteurs dans l'espace et dans le temps. Des biais subsistent, liés notamment au contrôle non total des géniteurs de truite de lac. La méthodologie employée peut cependant être préconisée par exemple dans le cas d'études préalables à des aménagements ou à l'installation de pièges dans des affluents de lac. 
Le présent travail constitue une première étape qui a permis de recueillir des premiers éléments de stratégie. La question reste posée de savoir si le déroulement de la fraie dans l'espace et dans le temps est optimal ou non, vis-à-vis de la production de truites de lac. La mise en place de pièges (montée et descente) permettrait de franchir une nouvelle étape.

\section{REMERCIEMENTS}

Les travaux présentés ci-dessus ont été menés dans le cadre d'une convention INRA-CSP (83-624 du 6/12/83) portant sur la truite de lac et d'une ATP-INRA consacrée aux écosystèmes lacustres. Nous tenons à remercier les propriétaires riverains du Redon, l'APP du Chablais Genevois, la DDA de Haute Savoie et les gardes-pêche associés.

\section{BIBLIOGRAPHIE}

ALM., 1949. Influence of heredity and environment on various forms of trout. Rep. Inst. Freshwater Res. Drott., 29, 29-34.

ARVIDSON G., 1935. Märkning av Laxöring i Vättern. Rep. Inst. Freshwater Res, Drott., 4, 1-16.

BAGLINIERE J.L., CHAMPIGNEULLE A. et NIHOUARN A., 1979. La fraie du saumon atlantique (Salmo salar L.) et de la truite commune (Salmo trutta L.) sur le bassin du Scorff. Cybium $3^{\ominus}$ série, $7,75-96$.

BAGLINIERE J.L., MAISSE G., LEBAIL P.Y. et PREVOST E., 1987. Dynamique de la population de truite commune (Salmo trutta L.) d'un ruisseau breton (France) : les géniteurs migrants. Acta Oecologica Oecol. Applic., $8: 201-215$.

BUTTIKER B. et MATTHEY G., 1986. Migration de la truite lacustre (Salmo trutta lacustris L.) dans le Lèman et ses affluents. Schweiz. Z. Hydrol., 48, 153-160.

BUTTIKER B., MATTHEY G., BEL J. et DURAND P., 1987. Age et croissance de la truite lacustre (Salmo trutta lacustris L.) du Léman Schweiz Z. Hydrol., 49, 316-328.

CHAMPIGNEULLE A., 1978. Caractéristiques de l'habitat piscicole et de la population de juvéniles sauvages de saumon atlantique (Salmo salar L.) sur le cours principal du Scorff (Morbihan). Thèse $3^{\text {e }}$ cycle, Biol. anim., Univ. Rennes, $132 \mathrm{p}$.

CHAMPIGNEULLE A., 1987. Etude sur la truite de lac et mises au point techniques. Rapp. Inst. Limnol. Thonon, 27, 1 vol., $10 \mathrm{p}$.

CUINAT R., 1974. Les exigences des poissons dans nos eaux courantes. Rap. Conseil Supérieur de la Pêche, $26 \mathrm{p}$.

EUZENAT G. et FOURNEL F., 1976. Recherches sur la truite commune (Salmo trutta L.) dans une rivière de Bretagne, le Scorff. I. Caractéristiques des populations de truite commune de la rivière Scorff et ses affluents. II. Premiers éléments d'une étude de la dynamique de population de truite commune. Thèse $3^{3}$ cycle Biol. Anim. Fac. Univ. Rennes, $213 p$.

GERDEAUX D., 1987. Note technique. Revue des méthodes d'estimation de l'effectif d'une population par pêches successives avec retrait. Programme d'estimation d'effectif par la méthode de CARLE et STRUB. Bull. Fr. Pêche Piscic., 304, 13-21.

GREELEY J.R., 1932, The spawing habits of brook, brown and rainbow trout and the problem of egg predators. Trans. Am. Fish. Soc., 62, 238-248.

HART P. J.B. et PITCHER T.J., 1969. Field trials of fish marking using a jet inoculator. J. Fish., Biol., 1, 383-385.

HELAND M., 1977. Recherches sur l'ontogénèse du comportement territorial chez l'alevin de truite commune (Salmo trutta L.). Thèse $3^{e}$ cycle. Biologie animale. Université de Rennes, $239 \mathrm{p}$.

HOBBS D.F., 1940. Natural reproduction of trout in New Zealand, its relation to density of populations. New Zealand Marine Dept. Fish. Bull., 8, $93 \mathrm{p}$.

LE BAIL P.Y., MAISSE G. et BRETON B., 1981. Détection des femelles de salmonidés en vittellogénèse. 1) Description de la méthode et mise en œuvre pratique. Bull. Fr. Piscic., 283, 79-88.

LELEK A., 1980. Les poissons d'eau douce menacés en Europe. Conseil de l'Europe, Strasbourg. Rapp. Sauvegarde de la Nature, 18, 1 vol., 277 p.

MAISSE G., BAGLINIERE J.L. et LEBAIL P.Y., 1987. Dynamique de la population de truite commune (Salmo trutta L.) d'un ruisseau breton (France). Les géniteurs sédentaires. Hydrobiologia, 148, 123-130.

MCNEIL W. and AHNELL W.H., 1964. Success of pink salmon spawning relative to size of spawning bed materials. US Fish and WildI. Serv. Spec. Scient. Rep. Fish., 469, 15 p. 
MELHAOUI M., 1985. Eléments d'écologie de la truite de lac (Salmo trutta L.) du Léman dans le système lac-affluent. Thèse Doct. $3^{\circ}$ Cycle, Univ. Pierre et Marie Curie, Paris VI, $127 \mathrm{p}$.

MUNRO W.R., BALMAIN K.H., 1956. Observations on the spawning runs of brown trout in the South Queich, Loch Leven. Freshwat. Salm. Fish. Res., 13, 17 p.

NIHOUARN A., 1983. Etude de la truite commune (Salmo trutta L.) dans le bassin du Scorff (Morbihan) : démographie, reproduction, migrations. Thèse Doct. $3^{\circ}$ cycle, Univ. Rennes, $64 \mathrm{p}$.

OTTAWAY E.M., CARLING P.A., CLARKE A. and READER N.A., 1981. Observations on the structure of brown trout (Salmo trutta L.) redds. J. Fish Biol., 19, 593-607.

RICHARD A., 1986. Recherches sur la truite de mer (Salmo trutta L.) en Basse Normandie : scalimétrie, sexage, caractéristiques biométriques, démographiques et migratoires. Thèse $3^{e}$ cycle, Fac. Sci. Univ. Rennes, 1 vol., 54 p.

RUHLE C., DEUFEL J., KEIZ G., KINDLE T., KLEIN M., LOFFLER H. et WAGNER B., 1984. Die Bodensee-Seeforelle. Probleme und Problemlösungen. Osterreichs Fisherei, 37, 272-307.

RUNNSTROM S., 1949. Control of trout migration by a fish ladder. Rep. Inst. Freshwater Res. Drottningholm, 29, 85-88.

RUNNSTROM S., 1952. The population of trout (Salmo trutta) in regulated lakes. Rep. Inst. Freshwater Res. Drottningholm, 33, 178-188.

RUNNSTROM S., 1957. Migration, age and growth of the brouw trout (Salmo trutta L.) in lake Rensjön. Rep. Inst. Freshwater Res., 38, 194-246.

SAKOWICZ St., 1961. Reproduction of the lake trout (Salmo trutta m. lacustris L.) from Wdyzdze lake. A. biologico-managing monograph of the lake trout form Wolzydzi Lake. Roczn. Nauk Roln., 93-D, 501-556.

SHIRWELL C.S. and DUNGEY R.G., 1983. Microhabitats chosen by brown trout for feeding and spawning in rivers. Trans. Am. Fish. Soc., 112, 355-367.

STUART T.A., 1953. Spawning migration, reproduction and young stages of loch trout (Salmo trutta L.). Sci. Invest. Freshwat. Salm. Fish. Res. Scotl., 5, 39 p.

STUART T.A., 1957. The migrations and homing behaviour of brown trout (Salmo trutta L.). Sci. Invest. Freshwat. Salm. Fish. Res. Scotl., 18, 27 p.

THORPE J.E., 1974. The movements of brown trout (Salmo trutta L.) in Loch Leven, Kinross, Scotland. J. Fish. Biol., 6, 153-180. 